gender, education level, history of angina, history of myocardial infarction, history of angioplasty, previous coronary artery bypass surgery, diabetes, current smoking status, hypercholesterolaemia and hypertension. Those who perceived themselves to be at higher risk of a future event were more likely to be female ( $p<0.001)$, have a higher education level $(p=0.02)$, aged between 44 and $59(p=0.02)$ and have a previous history of percutaneous coronary angioplasty $(\mathrm{p}=0.003)$.

Conclusions/Implications Despite the fact that the entire study sample were diagnosed with ACS, approximately one in five $(20 \%)$ perceived that they were less likely than other people of the same age to experience a heart attack in the future. These findings highlight the need for specific and targeted education of both men and women in Ireland with respect to the risks for heart disease and heart attacks.

\section{IMPACT OF CONTRAST-INDUCED NEPHROPATHY UPON SHORT AND LONG-TERM OUTCOMES OF PATIENTS WITH ST-SEGMENT ELEVATION MYOCARDIAL INFARCTION UNDERGOING PRIMARY PERCUTANEOUS CORONARY INTERVENTION}

doi:10.1136/heartjnl-2012-301877b.124

Sean Gallagher, ${ }^{*}$ S Hassan, D A Jones, M J Lovell, A Ahktar, A Kapur, C Knight, A Mathur, M M Yaqoob, A Wragg. Barts and the London NHS Trust, London, UK

Introduction Contrast induced nephropathy (CIN) is associated with adverse clinical outcomes, including prolonged hospitalisation and increased morbidity and mortality following elective invasive cardiac procedures. The impact of CIN following primary $\mathrm{PCI}$ (PPCI) for ST segment elevation MI (STEMI) remains poorly defined.

Aim To investigate the long-term prognostic implications of CIN following PPCI for STEMI.

Methods This is a retrospective observational registry study. Data were available upon 2224 patients undergoing PPCI for STEMI at a tertiary Cardiac centre between October 2003 and May 2010. CIN was defined as an increase in serum creatinine ( $>25 \%$ or $44.2 \mathrm{mmol} /$ 1) within 2 days of PPCI. The primary outcome measure was allcause mortality determined via Office of National Statistics data.

Results CIN was observed in 317 patients (14.3\%). Patients with CIN were older (69.3 vs 62.7, $\mathrm{p}<0.0001)$, more likely to be female (30.0\% vs $22.6 \% \mathrm{p}=0.004)$, had more vascular risk factors (including diabetes, hypertension and chronic kidney disease), had more previous MIs (17.7\% vs $11.7 \%, p=0.003)$, more multivessel CAD

Abstract 124 Table 1 Clinical characteristics of study cohort

\begin{tabular}{lccc}
\hline & No AKI (n=1907) & AKI (n=317) & Significance \\
\hline Age (yrs \pm SD) & $62.7 \pm 13.8$ & $69.3 \pm 1.8$ & 0.004 \\
Age $>75, \mathrm{n}(\%)$ & $404(21.2)$ & $128(40.4)$ & $<0.0001$ \\
Female, n (\%) & $431(22.6)$ & $95(30.0)$ & $<0.0001$ \\
Hypertension, n (\%) & $720(37.8)$ & $147(46.4)$ & 0.004 \\
Diabetes mellitus, n (\%) & $324(17.0)$ & $81(25.6)$ & $<0.0001$ \\
Hypercholestrolaemia, n (\%) & $1120(58.7)$ & $149(47.0)$ & $<0.0001$ \\
Smoking history, n (\%) & $554(29.1)$ & $98(30.9)$ & 0.500 \\
eGFR <60 mls/min, n (\%) & $355(18.6)$ & $105(33.1)$ & $<0.0001$ \\
Mean eGFR ( \pm SD) & $77.6 \pm 21.0$ & $75.1 \pm 31.4$ & 0.06 \\
Previous MI, n (\%) & $223(11.7)$ & $56(17.7)$ & 0.003 \\
Previous CABG, n (\%) & $52(2.7)$ & $12(3.8)$ & 0.296 \\
Previous PCI, n (\%) & $180(9.4)$ & $36(11.4)$ & 0.286 \\
EF $<50 \%$ & $613(32.1)$ & $165(42.1)$ & $<0.0001$ \\
Multivessel CAD, n (\%) & $774(40.6)$ & $175(55.2)$ & $<0.0001$ \\
Cardiogenic Shock, n (\%) & $88(4.6)$ & $43(13.6)$ & $<0.0001$ \\
\hline
\end{tabular}

(55.2\% vs $40.6 \%, \mathrm{p}<0.0001)$, and more LV dysfunction $(52.1 \%$ vs $32.1 \%, \mathrm{p}<0.0001$ ) (Abstract 124 table 1). Length of hospital stay ( 5 vs 2 days, $p<0.0001)$, 30 -day mortality ( $16.4 \%$ vs $2.0 \%$, $\mathrm{p}<0.0001)$ and 3 -year mortality $(22.4 \%$ vs $5.0 \%$, Log rank $\mathrm{p}<0.0001)$ (Abstract 124 figure 1) was worse in patients with CIN. CIN was an important independent predictor of all cause mortality (HR 2.71, CI 1.97 to 3.75). Other independent predictors of mortality included age $>75$ years (HR 1.81, CI 1.28 to 2.57), eGFR $<60 \mathrm{mls} / \mathrm{min}$ (HR 2.36, CI 1.69 to 3.31), cardiogenic shock (HR 3.98, CI 2.76 to 5.73 ), LV dysfunction (HR 1.82, CI 1.34 to 2.48) and multivessel CAD (HR 1.59, CI 1.15 to 2.20 ).

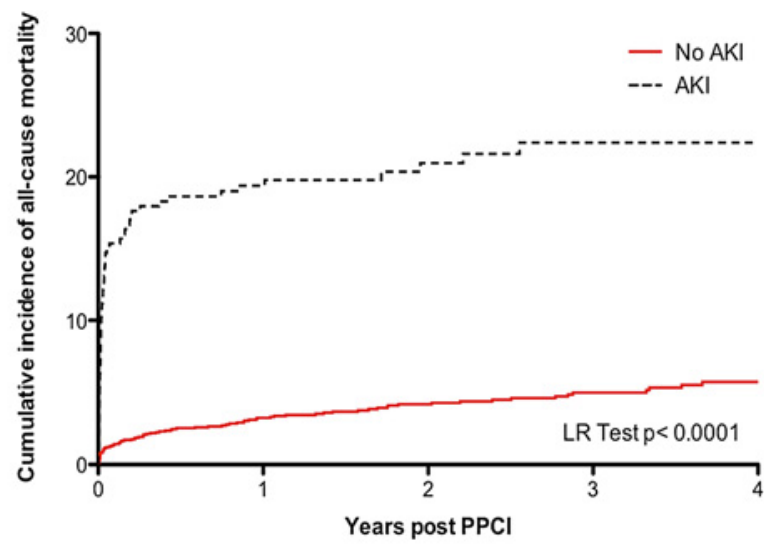

Numbers at Risk

No AKI 1907 1426 1034 656 360 AKI 319 205

133

58

Abstract 124 Figure 1 Effect of AKI upon all-cause mortality following PPCl for STEMI.

Conclusion CIN following PPCI for STEMI was associated with increased short and long-term mortality and increased length of hospital stay. Better strategies are needed to prevent CIN in high risk STEMI patients.

\section{MONOCYTE SUBPOPULATION COUNTS AND FUNCTIONAL CHARACTERISTICS PREDICT ADVERSE CLINICAL EVENTS POST ST ELEVATION MYOCARDIAL INFARCTION}

doi:10.1136/heartjnl-2012-301877b.125

${ }^{1}$ A A Ghattas, ${ }^{*} \mathrm{G}$ Y H Lip, ${ }^{2} \mathrm{H}$ Griffiths, ${ }^{2} \mathrm{E}$ Shantsila. ${ }^{1}$ Centre for Cardiovascular Sciences, University of Birmingham, Birmingham, UK; ${ }^{2}$ Schoolf of Life and Health Sciences Aston University, Birmingham, UK

Background Monocytes are implicated in the initiation of the atherosclerotic plaque through to plaque instability and rupture during presentation with an acute coronary syndrome (ACS). Little is known about the predictive role of monocytes on clinical outcome. We studied the role of the three phenotypically and functionally discrete monocyte subpopulations in predicting major adverse cardiac events (MACE)-defined as recurrent ACS, heart failure and death- following ST elevation myocardial infarction (STEMI).

Method STEMI patients treated with percutaneous revascularisation, were recruited in the first $24 \mathrm{~h}$ post-infarction. Peripheral blood monocyte subpopulations were enumerated and characterised using flow cytometry after staining for CD14, CD16 and CCR2. Phenotypically, monocyte subpopulations are defined as: CD14+ $+\mathrm{CD} 16-\mathrm{CCR} 2+$ (Mon1), CD14++CD16+CCR + (Mon2) and CD14+CD16++CCR2- (Mon3) cells. Functionally, monocyte subpopulation activation of nuclear factor $\kappa \mathrm{B}(\mathrm{NF} \kappa \mathrm{B})$ was analysed. Activation of $\mathrm{NF} \kappa \mathrm{B}$ was determined by flow cytometry as the mean 